\title{
Submillimeter View of Gas and Dust in the Forming Super Star Cluster in NGC 5253
}

\author{
Jean L. Turner ${ }^{1}$ \\ ${ }^{1}$ Department of Physics and Astronomy, UCLA, \\ Los Angeles, CA 90095-1547 USA \\ email: turner@astro.ucla.edu
}

\begin{abstract}
A giant molecular cloud has been detected surrounding the supernebula in NGC 5253, revealing details of the formation and feedback process in a very massive star cluster. "Cloud D" was recently mapped in CO $\mathrm{J}=3-2$ with the Submillimeter Array. The cloud surrounds a currently forming massive cluster of mass $\sim 10^{6} \mathrm{M}_{\odot}$, and luminosity $\sim 10^{9} \mathrm{~L}_{\odot}$. Cloud $\mathrm{D}$ is hot, clearly associated with the cluster, yet kinematically relatively quiescent. The dust mass is $\sim 15,000 \mathrm{M}_{\odot}$, for a gas-to-dust ratio of $\sim 50$, nearly an order of magnitude lower than expected for this low metallicity galaxy. We posit that enrichment by the cluster, leading to a stalled cluster wind, has created the unusual conditions in Cloud D. The absence of current mechanical impact of the young cluster on the cloud, in spite of the presence of thousands of O stars, may permit future generations of stars to form near the massive cluster.
\end{abstract}

Keywords. galaxies: individual (NGC 5253), galaxies: star clusters, galaxies: starburst, galaxies: dwarf

\section{Introduction}

The process by which the most massive star clusters form is not well understood. The closest massive clusters, such as those in the Galactic Center or R136 in the Large Magellanic Cloud, are relatively evolved and far from their initial molecular conditions. Clusters that are still embedded in their natal clouds can provide more direct information on the interaction of young clusters with their natal clouds, including star formation efficiency and the precise nature of cluster feedback on the clouds. The advantage of submillimeter observations in the study of young clusters is that one can simultaneously trace spectral lines of molecular gas as well as continuum emission from ionized gas and dust, to give a full picture of the gas, dust, and kinematics in young, forming clusters.

The massive cluster in NGC 5253 may be the closest example of a embedded cluster still in the process of formation. Despite having little gas and no disk, this dwarf spheroidal galaxy has formed hundreds of clusters in the recent past (Calzetti et al. 1997, 2015, Chandar et al. 2005, Harbeck et al. 2012, de Grijs et al. 2013). This unlikely environment is the location of the "supernebula", a bright and compact radio continuum source associated with a luminous HII region (Beck et al. 1996, Turner et al. 2000). The HII region core is $\sim 1 \mathrm{pc}$ in radius with an infrared luminosity of $\sim 10^{9} \mathrm{~L}_{\odot}$ at $\mathrm{D}=3.8 \mathrm{Mpc}$, and Lyman continuum rate of $\sim 7 \times 10^{52} \mathrm{~s}^{-1}$ (7000 O7 star equivalents) (Meier et al. 2002). The supernebula is embedded, with $\mathrm{A}_{\mathrm{v}} \sim 16$ (Turner et al. 2003, Alonso-Herrero et al. 2004). Most of the gas in NGC 5253 resides outside the galaxy, in a halo of HI filaments (Kobulnicky \& Skillman 2008; López-Sánchez et al. 2012) and in a molecular streamer of $\sim 200$ pc extent detected in CO (Turner et al. 1997). The streamer is infalling along the minor axis of the galaxy. No $\mathrm{CO}(1-0)$ and only weak $\mathrm{CO}(2-1)$ had been detected until 
recently at the location of the embedded SSC/supernebula (Turner et al. 1997; Meier et al. 2002; Miura et al. 2015).

Submillimeter Array observations of the $\mathrm{J}=3-2$ transition of $\mathrm{CO}$ and dust continuum in NGC 5253 have revealed the giant molecular cloud (GMC) associated with the supernebula and its embedded cluster. "Cloud D" is shown in Figure 1 (Turner et al. $2015)$. Cloud D is unusually bright in $\mathrm{CO}(3-2), 2.6$ times brighter than in $\mathrm{CO}(2-1)$. The emission is optically thin and the inferred gas temperature is $>200 \mathrm{~K}$, with densities of $\sim 5 \times 10^{4} \mathrm{~cm}^{-3}$. Galactic GMCs, even those actively forming stars, more typically have temperatures of $\sim 10-15 \mathrm{~K}$ on these scales. From its temperature alone, it is clear that Cloud D is closely associated with the young massive cluster.

To determine a star formation efficiency for the cluster we need a gas mass, which is difficult to determine for this strange cloud. From the $\mathrm{CO}(3-2) / \mathrm{CO}(1-0)$ ratio it is inferred that the emission is optically thin; by contrast, Galactic GMCs are optically thick in CO. Thus we cannot use the standard Galactic conversion factor, $X_{C O}$, which applies to optically thick and cooler Galactic clouds in the $\mathrm{CO}(1-0)$ transition. Cloud D is as yet undetected in $\mathrm{CO}(1-0)$. For optically thin emission, instead one counts emitted photons in the line and from that computes the total number of $\mathrm{CO}$ molecules from the partition function. However, to get to the total molecular mass requires a relative abundance of $\mathrm{CO}$ to $\mathrm{H}_{2}$, and this abundance depends on the chemistry. The chemistry is uncertain in Cloud D because of the unusually high temperature of the cloud, the high radiation fields, and the low metallicity of NGC 5253, $Z \sim 0.25 Z_{\odot}$ (Kobulnicky et al. 1997, López-Sánchez et al. 2007). Using the Galactic value of $[\mathrm{CO}] /\left[\mathrm{H}_{2}\right]=8.5 \times 10^{-5}$ we infer a total $\mathrm{H}_{2}$ mass of $M_{H_{2}}=5 \times 10^{4} \mathrm{M}_{\odot}$ (Turner et al. 2015); however, given the unusual properties of this cloud we have no confidence that the Galactic $\mathrm{CO}$ abundance ratio obtains here.

A less slippery number for the gas mass can be determined from subtracting the stellar mass from the dynamical mass of the cloud. The uncertainties are simpler and better constrained than the previous methods; it gives a total gas mass rather than an $\mathrm{H}_{2}$ mass, which is more appropriate for star formation efficiency. The mass of stars can be obtained from the Lyman continuum rate (Meier et al. 2002) and the 3.5 Myr age based on $\mathrm{Br} \gamma$ equivalent widths (Alonso-Herrero et al. 2004) and Wolf-Rayet signatures in abundances (Walsh \& Roy 1989, Kobulnicky et al. 1997, López-Sánchez et al. 2007). Calzetti et al. (2015) argue that dust emission affects the $\mathrm{Br} \gamma \mathrm{EW}$ and that the age of this cluster is closer to $1 \mathrm{Myr}$, and further divide the stars among two clusters, both within Cloud D. However, this young age would not explain the Wolf-Rayet signatures and local nitrogen enhancement in the region, so we favor an older age. For a $3.5 \mathrm{Myr}$ age, and assuming continuous star formation, the stellar mass based on STARBURST99 models is $M_{\text {stars }}=1.1_{-0.2}^{+0.7} \times 10^{6} \mathrm{M}_{\odot}$. The CO linewidth is $\sigma=9.2 \pm 0.6 \mathrm{~km} \mathrm{~s}^{-1}$; this will give an upper limit to the gravitational mass, which is $M_{v i r}=1.8_{-0.7}^{+0.2} \times 10^{6} \mathrm{M}_{\odot}$. The final gas mass is then $M_{v i r}-M_{\text {stars }}=7 \pm 4 \times 10^{5} \mathrm{M}_{\odot}$, giving a star formation efficiency $M_{\text {stars }} /\left(M_{\text {stars }}+M_{\text {gas }}\right) \sim 60 \%$ (Turner et al. 2015). This very high, record efficiency, not so different from the $75 \pm 50 \%$ inferred from $\mathrm{CO}(2-1)$ using $X_{C O}$ by Meier et al. (2002), bodes well for the cluster's survival.

\section{Cloud D: A Very Dusty Cloud}

An alternative path to the gas mass is through dust, but the dust mass of Cloud D is also strange and interesting. About half of the $72 \mathrm{mJy}$ of $870 \mu \mathrm{m}$ continuum emission, $34 \pm 14 \mathrm{mJy}$, is from dust; the rest is free-free emission from the ionized gas. Using the dust opacity of the Large Magellanic Cloud (Galliano et al. 2011), and a dust temperature of 


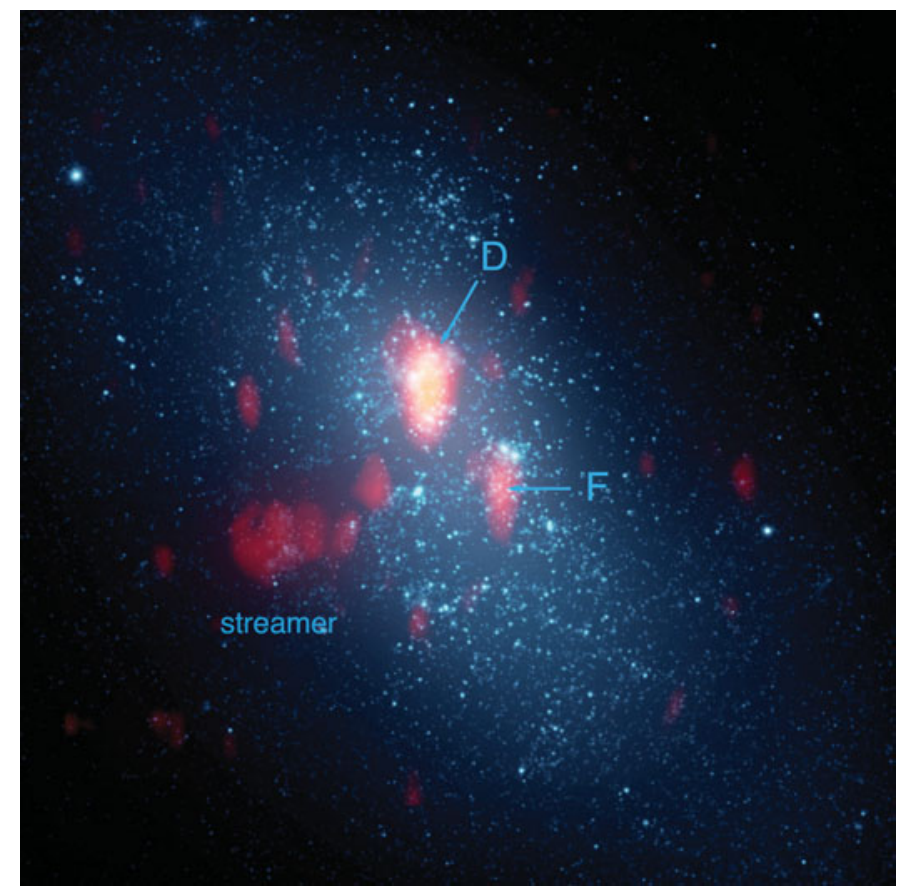

Figure 1. Molecular gas in NGC 5253. CO(3-2) emission overlaid on an HST image. The CO image is from the SMA, beam is 4 " $\mathrm{x} 2$ ". "Cloud D" is the brightest CO source near the center; also detected are another cloud, "F" and the "streamer", which is the extended filament to the east. Turner et al. 2015.

$46 \mathrm{~K}$ (Thronson \& Telesco 1986) we obtain $M_{d u s t}=1.5 \pm 0.2 \times 10^{4} \mathrm{M}_{\odot}$. If we scale the gas-to-dust ratio, GTD, of NGC 5253 to match its $Z \sim 0.25 Z_{\odot}$ metallicity, a common and reasonable assumption, we would estimate that $G T D \sim 500-700$, similar to values in the Magellanic Clouds, and this would give a dust-predicted gas mass of $M_{\text {gas }} \sim 10^{7} \mathrm{M}_{\odot}$. This is more than five times higher than the gas mass could possibly be, based on the dynamical mass of Cloud D. From the inferred gas mass we infer that instead GTD 50 in NGC 5253, a factor of three less than the Galactic GTD value (Turner et al. 2015).

The reason that NGC 5253 has ten times more dust than expected may be enrichment by the cluster stars, metals that are as yet trapped within the natal cloud. STARBURST99 models for clusters of this luminosity that are more than $3 \mathrm{Myr}$ of age can produce 30,000 $\mathrm{M}_{\odot}$ of metals, twice the dust mass that is seen. So it is not surprising, it is even expected, that enrichment is taking place.

The dust enrichment has potential implications for the evolution of the cluster and its cloud, and the nature of feedback. Cloud D is surprisingly quiescent; its linewidth of $\sim 9 \mathrm{~km} \mathrm{~s}^{-1}$ is not far from the value expected for a cloud of its $25 \mathrm{pc}$ size based on the Galactic size-linewidth relation. Yet its high temperature and precise positional and kinematic coincidence with the massive cluster indicates a close association of the two. Where is the feedback from the 7000 O stars?

Silich et al. (2004) and Tenorio-Tagle et al. (2007) suggest that enrichment by cluster stars creates a dusty environment that can enhance gas cooling and stall a cluster wind. Based on numerical modeling of the gas dynamics they find that for a cluster of the luminosity exciting the supernebula in NGC 5253, an enrichment of $1.5 Z_{\odot}$ is sufficient to stall a wind, trapping the enriched products of stellar evolution within the cloud. From the mass of dust it would appear that the enrichment of the gas in Cloud D is roughly 
twice this amount. The stalled wind model could explain both the quiescence of Cloud D and its dustiness. Given the relative quiescence of this cloud, so similar to Galactic GMCs, it would seem that conditions are ripe for the formation of even more stars at this location, given an influx of gas. This is a very interesting suggestion (Silich et al. 2004; Tenorio-Tagle et al. 2007) in light of the fact that multiple generations are observed to be present in globular clusters (see also R. Wünsch this volume).

\section{Conclusions}

Submillimeter Array maps of $\mathrm{CO}(3-2)$ and $870 \mu \mathrm{m}$ dust continuum emission from Cloud $\mathrm{D}$ associated with the young, embedded massive cluster exciting the supernebula in NGC 5253 allow the determination of dynamical mass, gas mass, and dust mass for this cloud, and allow us to define the interaction of the cluster and its surroundings.

1) The molecular gas in Cloud $\mathrm{D}$ is hot; the $\mathrm{CO}(3-2) / \mathrm{CO}(2-1)$ line ratio suggests that the temperature is unusually warm, $>200 \mathrm{~K}$. The cloud is clearly heated by the cluster.

2) The $\mathrm{H}_{2}$ mass obtained from the line intensity of the optically thin $\mathrm{CO}$ emission is low, particularly compared to the dust mass. This is probably due to the $[\mathrm{CO}] /\left[\mathrm{H}_{2}\right]$ abundance ratio, which is highly uncertain in this unusually warm and highly irradiated cloud. Instead we obtain a gas mass by subtracting the stellar mass, based on the Lyman continuum rate and STARBURST99 models and a 3.5 Myr age, from the dynamical mass based on the CO size and linewidth of Cloud D. The gas mass of $7 \times 10^{5}(\mathrm{D} / 3.8 \mathrm{Mpc})^{2}$ gives a record high star formation efficiency of $60 \%$.

3) The dust mass is high for the metallicity of the galaxy: gas-to-dust radio is 50:1, sub-solar, much lower than the 500-700 value expected based on the low metallicity of NGC 5253. We posit that the dust mass is almost entirely produced by the stars of the cluster; STARBURST99 models predict twice this yield in metals for a cluster of this luminosity and 3.5 Myr age, so this model is entirely plausible.

4) The numerical models of Silich et al. (2004) predict that a cluster wind will catastrophically cool and stall if the gas is enriched to $1.5 Z_{\odot}$ for the supernebula cluster in NGC 5253. This condition appears to be met. This could explain why, even in the presence of $7000 \mathrm{O}$ stars and a high cloud temperature, the width of the CO line, 9.2 $\mathrm{km} / \mathrm{s}$, roughly that expected for a quiescent cloud of its size based on the Galactic sizelinewidth relation. Cluster feedback of the mechanical variety appears to be inhibited in this cluster, which could allow further accretion and future generations of stars to form in this cluster.

\section{Acknowledgements}

JLT would like to acknowledge S. Michelle Consiglio for helpful discussions, and the support of NSF Grant AST-1515570.

\section{References}

Alonso-Herrero, A., Takagi, T., Baker, A. J., Rieke, G. H., Rieke, M. J., Imanishi, M., \& Scoville, N. Z. 2004, ApJ, 612, 222

Beck, S. C., Turner, J. L., Ho, P. T. P.., Lacy, J. H., \& Kelly, D. M. 1996, ApJ, 457, 610

Calzetti, D., Meurer, G. R., Bohlin, R. C., Garnett, D. R., Kinney, A. L., Leitherer, C., \& Storchi-Bergmann, T. 1997, AJ, 114, 1834

Calzetti, D., Johnson, K. E., Adamo, A., et al. 2015, arXiv:1508.04476

Chandar, R. et al. Leitherer, C., Tremonti, C. A., Calzetti, D., Aloisi, A., Meurer, G. R., , de Mello, D. 2005, ApJ, 628, 210

de Grijs, R., Anders, P., Zackrisson, E., \& Östlin, G. , 2013 MNRAS, 431, 2917 
Galliano, F., Hony, S., Bernard, J.-P., et al. 2011, A\&A, 536, A88

Harbeck, D., Gallagher, J., \& Crnojević, D. 2012, MNRAS, 422, 629

Kobulnicky, H. A. \& Skillman, E. D. 2008, AJ, 135, 527

Kobulnicky, H. A., Skillman, E. D., Roy, J.-R., Walsh, J. R., \& Rosa, M. R. 1997, ApJ, 477, 679

López-Sánchez, Á. R., Esteban, C., García-Rojas, J., Peimbert, M., \& Rodríguez, M. 2007, ApJ, 656,168

López-Sánchez, Á. R., Koribalski, B. S., van Eymeren, J., Esteban, C., Kirby, E., Jerjen, H., \& Lonsdale, N. 2012, MNRAS, 419, 1051

Meier, D. S., Turner, J. L., \& Beck, S. C. 2002, AJ, 124, 877

Miura, R. E., Espada, D., Sugai, H., Nakanishi, K., \& Hirota, A. 2015, PASJ, 67, LL1

Silich, S., Tenorio-Tagle, G., \& Rodríguez-González, A. 2004, ApJ, 610, 226

Tenorio-Tagle, G., Wünch, R., Silich, S., \& Palous, J. 2007, ApJ, 658, 1196

Thronson, H. A., Jr. \& Telesco, C. M. 1986, ApJ, 311, 98

Turner, J. L., Beck, S. C., \& Ho, P. T. P. 2000, ApJ, 532, L109

Turner, J. L., Beck, S. C., \& Hurt, R. L. 1997, ApJL, 474, L11

Turner, J. L., Beck, S. C., Crosthwaite, L..P., Larkin, J. E., McLean, I. S., \& Meier, D. S. 2003, Nature, 423, 621

Turner, J. L., Beck, S. C., Consiglio, S. M., Ho, P. T. P., Kovács, A., Meier, D. S., \& Zhao, J.-H. 2015, Nature, 519, 331

Walsh, J. R. \& Roy, J.-R. 1989, MNRAS, 239, 297

\section{Discussion}

BOESGAARD: How did you get the metallicity?

TURNER: The overall metallicity of NGC 5253, $Z \sim 0.25 Z_{\odot}$, is from observations of the surrounding HII regions. Optical emission lines cannot be detected from the supernebula directly, which is embedded in Cloud D. The extinction is about 16 magnitudes in the visual. The gas-to-dust ratio is directly computed from the gas mass of Cloud D and observed dust mass. 